\section{Propuesta para la documentación y clasificación de las técnicas constructivas romanas}

\section{Proposal for recording and classifying the Roman building techniques}

Antonio Pizzo*

Università di Roma-La Sapienza. Dipartimento RADAAR

\begin{abstract}
Resumen
Este artículo pretende formalizar y redefinir el análisis de las técnicas constructivas de un contexto arquitectónico de época romana, planteando para ello documentar una serie de parámetros que permitan comprender y homogeneizar el proceso de clasificación y con el objetivo futuro de una puesta en común de los datos registrados. Este camino, escasamente utilizado por la arqueología clásica, necesita, en nuestra opinión, de un evidente proceso de generalización en el ámbito de la Arqueología de la Arquitectura que, sin embargo, no termina de difundirse en los análisis arquitectónicos de las construcciones romanas. En este sentido, se presenta una ficha-tipo que facilita un amplio abanico de posibilidades contempladas a lo largo de varias experiencias en contextos territoriales diferentes.

El sistema de registro propuesto se plantea para la primera etapa de documentación y clasificación de los aspectos técnicos relacionados con la arquitectura de época romana, considerándose el mismo como un sistema abierto a la inserción de nuevos campos según los territorios y materiales analizados.
\end{abstract}

Palabras Claves: Técnicas constructivas romanas, documentación, clasificación, procesos constructivos.

\section{Abstract}

This paper intends to formalize and redefine the building techniques analysis of a roman architectural context by trying to record a group of parameters that allow understanding and homogenizing the classifying process. This has the future aim of getting a common proposal of the recorded facts. According to us, this way, scarcely used by the Classical archaeology, requires a generalization process which is clear within the Archaeology of Architecture but not enough known within the architectural analysis of Roman buildings. Regarding this, a type recording sheet is shown. This one enables a wide range of possibilities watched throughout different experiences in different territorial contexts.

The recording system here exposed is proposed for the first recording and classifying phase of the technical aspects related to Roman architecture, being an open system where new fields regarding territories and materials may be whenever introduced.
\end{abstract}

Keywords: Roman building techniques, recording, classification, building processes.

\section{INTRODUCCIÓN Y ANTECEDENTES 1}

El estudio de las técnicas constructivas de época romana vuelve a cobrar cierta atención en los últimos años, debido fundamentalmente a una serie de iniciativas surgidas de forma independiente en Siena ${ }^{2}$ y Mérida ${ }^{3}$, orientadas a crear una serie de nuevas tipologías con un cambio sustancial respecto a la visión estilística tradicional.

El análisis de las técnicas constructivas de época romana ha sufrido la corriente de estudios estilísticoscomparativos, basada en una metodología empírica orientada hacia la búsqueda de relaciones sistemáticas entre técnicas de edificios pertenecientes a épocas históricas y contextos territoriales muy distintos. Este planteamiento ha llevado a la creación de análisis diacrónicos de las técnicas, fundados casi exclusivamente en las connotaciones formales de los paramentos. Los caracteres estilísticos se han identificado, en la mayoría de los casos, con una cronología determinada, perdiendo de vista el fenómeno de la coexistencia de técnicas muy distintas entre ellas o, en sentido opuesto, la presencia de técnicas similares pertenecientes a épocas diferentes, casi siempre en favor de la búsqueda de «un progressivo avvicinamento a un momento apogeico, seguito da un graduale allontanamento dal presunto miglior costruire» ${ }^{4}$.

La tradición de estudios sobre la arquitectura romana ha intentado, durante muchos años, asociar la regularidad de los paramentos externos con las capacidades de los constructores y con la estética del edificio, distinguiendo, así, una serie de periodos con formas constructivas mejores, respecto a otras que se alejan de los cánones estéticos establecidos.

La perspectiva de los nuevos análisis citados, vinculados con el examen de la tecnología edilicia romana, se aleja de la visión de las técnicas constructivas como un elemento más de un estilo arquitectónico, combinación de reglas formales en relación con una cronología específica y preestablecida, para abrirse, en general, a una posición

\footnotetext{
1 Agradezco en general las indicaciones aportadas a este artículo por los evaluadores externos de la revista y, específicamente, algunas propuestas de enriquecimiento del sistema de registro y documentación de las técnicas constructivas.

${ }^{2}$ Camporeale 2004; Pais 2008.

${ }^{3}$ Pizzo 2010. Los resultados de estos trabajos, vinculados con la realización de tesis doctorales han generado la concesión de una Acción Integrada (Ref: HI2007-0236) entre el Instituto de Arqueología-Mérida y el Dipartimento di Archeologia e Storia delle Arti de la Università di Siena. Un estudio técnicoconstructivo se ha iniciado en los últimos meses en Tarragona, sobre los complejos monumentales del recinto superior de la ciudad, el concilio provincial y el circo. En este estudio se emplean y actualizan las premisas metodológicas desarrolladas en el análisis de la arquitectura pública de Augusta Emerita.

${ }^{4}$ Cagnana, A. 1994: pp. 39-40
} 
analítica que atienda a los elementos formales de los paramentos en relación con las dinámicas productivas, los tiempos y la organización del trabajo, los sistemas de aprovisionamiento de los materiales, etc.

En la arqueología clásica española, el grupo de investigación del Departamento de Prehistoria y Arqueología de la Universidad Autónoma de Madrid, dirigido por M. Bendala, ha desarrollado algunos procedimientos de sistematización del registro de las técnicas constructivas en trabajos efectuados sobre varias ciudades de la Bética ${ }^{5}$ de la Lusitania ${ }^{6}$.

Sin embargo, la nueva orientación metodológica de estos estudios ha tenido como punto de referencia las experiencias llevadas a cabo por la Arqueología de la Arquitectura y el interés de esta disciplina hacia la importancia del análisis de los aspectos constructivos como paso previo al conocimiento de los edificios.

Desde el inicio ${ }^{7}$, la Arqueología de la Arquitectura ha definido la importancia del estudio de las técnicas constructivas $^{8}$, considerando fundamental la necesidad de disponer de tipologías a escala regional y subregional ${ }^{9}$ y organizando la formación de instrumentos tipológicos y cronológicos a escala territorial ${ }^{10}$, como base del conocimiento cuantitativo de los contextos construidos ${ }^{11}$.

Originariamente, en este ámbito, el problema de las técnicas constructivas ha sido abordado respectivamente desde una perspectiva teórica y metodológica por $\mathrm{T}$. Mannoni ${ }^{12}$ y R. Parenti ${ }^{13}$. En la fase de consolidación del método estratigráfico aplicado a los edificios históricos, se ha considerado la técnica edilicia como un indicador fundamental para la definición de la cronología absoluta ${ }^{14}$, insertando el registro de la misma en una praxis de documentación más o menos analítica, dependiendo de la complejidad de las estructuras. La peculiaridad del método de estudio de la técnica constructiva, tal como se ha formulado en el ámbito de la Arqueología de la Arquitec-

${ }^{5}$ Roldán Gómez, L. 1987, 1992, 1994.

${ }^{6}$ Durán Cabello, R. 1991-1992, 2004; Bendala Galán, M. 1992, Bendala, M. Durán, R. 1994, Bendala, M. - Rico, C. Roldán, L. (Eds.) 1998; Rodríguez Gutiérrez, O. 2004.

${ }^{7}$ Mannoni, T. 1974: pp. 291-300.

${ }^{8}$ Caballero Zoreda, L. 1987: pp. 13-58.

${ }^{9}$ Brogiolo, G.P. 1996: p. 12.

10 Existen, en este sentido, trabajos puntuales relacionados con proyectos concretos de creación de atlantes de técnicas constructivas a escala regional y local. Véase por ejemplo: Mannoni, T. 1974: pp. 291-300; Parenti, R. 1988a: pp. 249-279; 1988b: pp. 280-304; Brogiolo; G.P. - Zonca, A. 1989: pp. 37-44; Caballero Zoreda 1999: pp. 221-233.

${ }^{11}$ Utrero Agudo, M.Á. 2008: p. 101.

${ }^{12}$ Mannoni, T. 1997: pp. 15-24.

${ }^{13}$ Parenti, R. 1988b: pp. 280-304.

${ }^{14}$ Parenti, R. 1988a: p. 249. tura, se centra en una estrecha vinculación con la estratigrafía de los paramentos y la individualización previa de las unidades estratigráficas ${ }^{15}$. Este reconocimiento facilita no solamente la posibilidad de comprender la evolución diacrónica del edificio, sino también la sucesión relativa de las distintas formas y modalidades constructivas.

T. Mannoni en el año $1984^{16}$ y R. Parenti en el $1988^{17}$ han establecido una serie de factores para la datación de las técnicas constructivas que prescinden, teóricamente, de diferencias entre periodos históricos y tipología de edificios. Los indicadores cronológicos se han dividido en fuentes indirectas y directas; las primeras asociadas a las fuentes históricas, cartográficas, iconográficas; las segundas se distinguen además en «relativas» (típicas de la arqueología estratigráfica) o «absolutas» (informaciones contenidas en los materiales o en elementos estructurales que forman el edificio) ${ }^{18}$. Con respecto a los sistemas de datación de las técnicas constructivas se insistía, ya a partir de los años 80 del siglo pasado, en el reconocimiento de «las claves cronológicas locales», con la intención de definir su variabilidad espacial y la relación con los factores naturales (diversidad de las fuentes de aprovisionamiento del material) o antrópicos (secuencias tipológicas y variaciones técnicas $)^{19}$.

Estas indicaciones se han consolidado en el estudio del mundo de la construcción medieval mediante un proceso básico definido por una serie de parámetros de clasificación que caracterizan, generalmente, la forma de construir una estructura ${ }^{20}$ :

- el tipo de material de construcción (litotipos),

- grado y tipo de elaboración empleado en la preparación del material,

- tipo de aparejo,

- dimensiones de los elementos constructivos,

- técnicas de acabado de los materiales,

- tipos de mortero.

En general, en el ámbito especifico de las técnicas de construcción se ha dado un amplio margen de maniobra al estudio de periodos históricos muy diferentes, con una serie diversificada de líneas de investigación que coinciden en la individualización de «dónde» se pueden encontrar

\footnotetext{
${ }^{15}$ Parenti, R. 1988a: p. 249.

${ }^{16}$ Mannoni, T. 1984: pp. 396-403.

${ }^{17}$ Parenti, R. 1988a: p. 249-279.

${ }^{18}$ Mannoni, T. 1984: p. 397.

${ }^{19}$ Mannoni, T. 1984: p. 399

${ }^{20}$ Parenti, R. 1988b: p. 282.
} 
determinadas técnicas, "cómo" se construía en un territorio concreto y «cuándo» se genera una forma constructiva, dependiendo de una menor o mayor duración y transmisión de los conocimientos técnicos acumulados ${ }^{21}$.

A pesar de estas primeras intenciones, se generó una pausa en la discusión de cuestiones metodológicas sobre el análisis técnico de los aparejos, con una dedicación casi exclusiva a los detalles de las superficies y los revestimientos, elementos escasamente presentes entre los restos arquitectónicos de época romana.

Mientras que, en general, se estancaba el interés hacia la creación de catálogos regionales de técnicas constructivas, en España se difundía la Arqueología de la Arquitectu$\mathrm{ra}^{22}$ y se evaluaba la importancia de estos estudios ${ }^{23}$, con relevantes aportaciones muy recientes ${ }^{24}$.

En los últimos años se ha recuperado, en este ámbito, el retraso en la aplicación de las metodologías citadas, fundamentalmente con las actividades de unas serie de grupos de investigación operantes en ámbitos territoriales diferentes ${ }^{25}$. En la Arqueología de la Arquitectura se ha producido un cambio ${ }^{26}$ respecto a los estudios limitados a la comprensión histórica de un edificio y se ha planteado una investigación rigurosa respecto a monumentos emblemáticos en relación con la historia de una ciudad o de una región ${ }^{27}$.

Con el desarrollo de estas experiencias y las abundantes aplicaciones de esta metodología a construcciones de diferente tipología es posible presentar, actualmente, un panorama de investigación más amplio y rico de matices que resulta fundamental para el desarrollo de nuestra propuesta de clasificación vinculada a las técnicas constructivas de época romana. Estas novedades permiten ampliarla con otros aspectos vinculados a los significados contenidos en las informaciones técnicas presentes en la arquitectura antigua y complementar los objetivos tradicionales de la disciplina (análisis estratigráfico-técnico) con

\footnotetext{
${ }^{21}$ Véase, en este sentido, la importante contribución de Parenti, R. 1994: pp. 2537.

${ }^{22}$ Caballero Zoreda, L. 1996: pp. 55-74; Caballero Zoreda, L. - Fernández Mier, M. 1997; pp. 147-158; en general véase Caballero Zoreda, L. - Escribano Velasco, C. 1996.

${ }^{23}$ Caballero Zoreda, L. 1999: pp. 221-233.

${ }^{24}$ Sánchez Zufiaurre, L. 2007.

${ }^{25}$ Se recuerdan, en este sentido, las investigaciones del Servei de Catalogació i Conservació de Monuments de la Diputación de Barcelona; el Centro de Estudios Históricos del CSIC y el Departamento de Arqueología de la Universidad de Vitoria. Véase Caballero Zoreda, L. - Fernández Mier, M. 1997; pp. 147158.

${ }^{26}$ Pizzo, A. 2003: pp. 316-317.

${ }^{27}$ Azkarate Garai-Olaun, A. 2002: pp. 55-71; en general Azkarate, A. - Cámara, L. - Lasagabaster, J.A. - La Torre, P. 2001
}

las exigencias y los objetivos de las ciencias históricas (contexto espacial-cronológico).

\section{CLASIFICACIÓN Y TIPOLOGÍA DE LAS TÉCNICAS CONSTRUCTIVAS DE ÉPOCA ROMANA: CUESTIONES GENERALES 28}

La base del trabajo de las ciencias históricas se fundamenta en dos operaciones esenciales (también en el caso de los que refutan reflexiones teóricas previas) - la clasificación y la tipología - presentes, en distinta medida, en la totalidad de los estudios que tratan de ámbitos productivos.

A.M. Sestieri, ha resumido, eficazmente, los distintos procesos de clasificación y la creación de tipologías a partir de distintas experiencias llevadas a cabo en las arqueologías europeas en las últimas décadas: «La clasificación de los materiales arqueológicos (incluyendo las estructuras de un edificio como un elemento más de la cultura material) consiste en el reconocimiento de la presencia recurrente de elementos técnicos, formales y dimensionales en los materiales manufacturados, preliminar al estudio de su contexto o de su inserción en una escala geográfica y cronológica en sentido amplio $»^{29}$. Esta definición recoge una regla general. En el caso de la clasificación de las técnicas constructivas de la arquitectura de época romana, se añade a los discriminantes de la clasificación el elemento funcional, en una estricta combinación con la forma, las dimensiones y la técnica de cada una de las estructuras analizadas. Con esta finalidad, en general, es útil dividir los edificios en el conjunto de elementos estáticos que los componen y que permiten, progresivamente, su construcción (desde los sistemas de cimentación hasta las cubiertas).

En cambio, "la tipología de los materiales arqueológicos tiende a reconocer diferencias formales sistemáticas y culturalmente significativas entre los objetos, como parte de la reconstrucción de conjunto de las comunidades que los produjeron y utilizaron ${ }^{30}$.

En el análisis de la arquitectura romana, la sistematización tipológica se realiza con el empleo de la nomenclatura tradicional de las técnicas constructivas (opus quadratum, incertum, mixtum, testaceum, caementicium, etc.), visto que las características generales de estas clases se pueden identificar, formalmente, con el resto de técnicas presentes en territorios muy distintos.

\footnotetext{
28 Los mismos conceptos se han empleado en el estudio de las técnicas constructivas de la arquitectura pública de Augusta Emerita, recientemente publicado. Véase en general Pizzo, A. 2010.

${ }^{29}$ Sestieri, A. M. 2001: p. 61.

${ }^{30}$ Sestieri, A. M. 2001: p. 61.
} 
En síntesis, para aprovechar al máximo las informaciones recopiladas en la catalogación, es necesario precisar un sistema de elaboración de los datos que, empezando por la fase de registro, alcance las cuestiones principales de la problemática tratada. Esta sistematización se puede obtener exclusivamente con la tipología, entendida como la ordenación de un conjunto de objetos (técnicas, en nuestro caso) desde los que el investigador deriva "delle inferenze relative a dei fatti che non sono contenuti nella rappresentazione iniziale di questi oggetti ${ }^{31}$.

Sin embargo, tras la realización de diferentes trabajos, creemos que el paso de la clasificación a la tipología (entendida como instrumento de definición de leyes formales y culturales que se repiten), es, todavía, demasiado corto. A pesar del reconocimiento de una cultura arquitectónica que se implanta y perdura con la historia de las ciudades, resulta difícil extrapolar agrupaciones a gran escala que, en el ámbito de una óptica constructiva muy estandarizada permitan vincular tipos o variantes a grupos técnicos específicos. En este sentido, la aparente homogeneización morfológica de los aparejos (presente todavía en la tradición de nuestros estudios) deriva de la limitación misma de los trabajos existentes.

Es necesario, en nuestra opinión, recuperar un planteamiento más específico vinculado con el estudio de las técnicas constructivas, centrado en el reconocimiento de las variaciones formales que dependen de un significado específico, con el deseo de extender este planteamiento a otros contextos, sin desistir de registrar las más pequeñas variables técnicas, y con la perspectiva de asociar, en el futuro, nuevos registros a nuevos factores disociados respecto al ámbito puramente formal.

\section{FUNDAMENTOS PARA LA CLASIFICACIÓN DE LAS TÉCNICAS CONSTRUCTIVAS ROMANAS}

Con el objetivo de evitar el análisis estilístico se ha creído necesario estructurar una propuesta de estudio de las técnicas constructivas, sobre la base de una amplia serie de elementos condicionantes, de tipo morfológico, productivo y tecnológico.

La gran variedad de los materiales empleados en la construcción de las distintas colonias dispersas en un territorio de proporciones desmesuradas exige, en nuestra opinión, un conocimiento especifico de los mismos a escala microterritorial y regional. Los modelos arquitectónicos que se implantan y transmiten en un territorio más o menos amplio durante el periodo romano, necesitan de

${ }^{31}$ Gardin, J.C. 1979: p. 116; Pucci, G. 1983: p. 286. una correcta clasificación orientada al conocimiento de los contextos de aprovisionamiento de los materiales o al desarrollo y la afirmación de determinadas técnicas respecto a otras. En este sentido, es evidente la imposibilidad de considerar una técnica constructiva simplemente como un detalle formal del edificio.

Con esta propuesta es necesario aceptar la definición de una técnica edilicia como el fruto de un ciclo productivo complejo ${ }^{32}$, basado en una serie de operaciones en relación con:

- el aprovisionamiento del material constructivo,

- la transformación del material en elemento funcional para el mecanismo estático del edificio,

- su colocación de acuerdo a un determinado conocimiento tecnológico.

Para la comprensión de los distintos procesos que llevan a la reconstrucción del ciclo de producción de una determinada técnica es preciso individualizar, en las estructuras, una serie de elementos que se definen, sintéticamente $^{33}$ :

- los componentes de las estructuras.

- las técnicas de transformación de los materiales desde la extracción hasta la construcción.

- las modalidades de edificación de las estructuras.

La cantidad de documentación que se obtiene con el trabajo de campo realizado en los edificios romanos exige, en nuestra opinión, la creación de una base de datos informatizada para el registro de las características técnicas de la arquitectura. Los contenidos del sistema deberían coincidir con una serie de registros que permita consultar, actualizar, ordenar y gestionar una serie de datos de carácter textual, gráfico y fotográfico. Nuestra propuesta se articula en la definición de seis niveles distintos que corresponden a las diferentes etapas de la investigación:

1) Un contenedor general sobre el «edificio» debería sintetizar las características principales, incorporando una descripción, un resumen de los detalles relativos a la estratigrafía y a la técnica constructiva, visualizando la bibliografía y la documentación existente. Desde este primer bloque de la base de datos debería ser posible consultar la totalidad de la información almacenada, con la

\footnotetext{
${ }^{32}$ Mannoni, T. - Giannichedda, E. 1996: pp. 98 ss.

${ }^{33}$ Mannoni, T. 1994; Brogiolo; G.P. 1996: p. 12.
} 
conexión directa a los siguientes bloques informatizados. En este mismo apartado resultará útil insertar un análisis historiográfico de los edificios, orientado a la búsqueda de referencias y representaciones gráficas antiguas, fundamentales para el conocimiento de la historia de las estructuras y para la individualización de elementos perdidos de la arquitectura conservada, posibles restauraciones o alteraciones de las fábricas.

2) En un segundo contenedor se ilustrará de forma específica los resultados de los "análisis estratigráficos» previos realizados en el edificio, ofreciendo, fundamentalmente, información sobre las cronologías relativas existentes entre técnicas diferentes o similares.

En los análisis estratigráficos se evidenciará un cuadro de visualización sintético de las relaciones estratigráficas entre las unidades documentadas, reflejando las relaciones de coetaneidad, posterioridad y anterioridad, evitando la complejidad de las relaciones físicas y facilitando, así, la asociación entre unidades estratigráficas y técnicas constructivas.

Las experiencias efectuadas en diferentes contextos nos han indicado la posibilidad de diferenciar el grado de profundidad del análisis estratigráfico, organizándolo según la complejidad de los edificios, en tres distintos niveles de actuación. Los niveles del análisis estratigráfico se han establecido en:

$\checkmark$ Análisis estratigráfico completo, siguiendo los patrones establecidos por las experiencias llevadas a cabo desde la Arqueología de la Arquitectura.

$\checkmark$ Análisis estratigráfico breve (limitado a la lectura rápida de las intervenciones o reformas que permitan establecer una cronología relativa entre técnicas constructivas o variantes diferentes).

$\checkmark$ Análisis estratigráfico parcial (limitado a la relación entre distintos cuerpos de fábrica del edificio, con el objetivo de leer el proceso de edificación y las modalidades de la construcción y las fases de la obra).

3) Los detalles del análisis técnico de los distintos elementos constructivos que conforman el edificio se recogerán en un bloque central de la base informatizada, empleando una serie de criterios que permitan abordar un examen distinto respecto al estudio de las connotaciones formales tradicionales.

En este caso, se plantea un apartado de datos generales que vincule las características técnicas con el edificio, en una óptica estratigráfica y estática; otro que analice los tipos de aparejos; un tercero para los tipos de materiales; y un último para el estudio de los factores que determinan un proceso de construcción.

Este contenedor servirá, además, para confeccionar el prototipo de ficha de campo, objetivo principal de esta propuesta. Los siguientes apartados permiten consultar la documentación existente sobre un edificio. En este sentido es útil contemplar la existencia de tres bloques distintos:

4) La bibliografía general y específica existente sobre el edificio objeto del análisis.

5) La documentación planimétrica relacionada.

6) La documentación fotográfica antigua y reciente.

La estructura de estos contenedores informatizados refleja las observaciones generales sobre un contexto construido, la definición de las características especificas de las técnicas de edificación y las distintas combinaciones de técnicas y materiales que determinan su cultura arquitectónica.

Del mismo modo, el sistema tiene que ser abierto y adaptarse a las distintas novedades que aparezcan durante el trabajo de documentación, a las diferentes variaciones en el registro de las técnicas constructivas o a la supresión e inserción de campos que no encuentran relaciones con nuestra propuesta específica.

\section{LA DOCUMENTACIÓN DE LAS TÉCNICAS CONSTRUCTIVAS ROMANAS}

Una de las principales problemáticas evidenciadas en el desarrollo de nuestros primeros trabajos de investigación ha sido la dificultad de documentar gráficamente las variantes de las técnicas constructivas, operación indispensable para un análisis arqueológico correcto.

En los últimos años, debido a los altos costes de la técnica fotogramétrica tradicional, la investigación arqueológica se orienta hacía la experimentación y la utilización de técnicas alternativas que puedan sustituir la documentación gráfica de los edificios realizadas con el levantamiento por medio de la fotogrametría arquitectónica.

Una opción que está manifestando validez y fiabilidad, desde el punto de vista de las representaciones bidimensionales de las fábricas de los edificios, es la ortorectificación fotográfica con apoyo topográfico. El dibujo de las variantes constructivas, se obtiene, así, mediante una serie de dibujos realizados en aplicaciones Cad, sobre los fotoplanos correspondientes corregidos.

La creación de un registro de imágenes digitales tomadas a corta distancia hace posible, además, una 
primera descomposición de los edificios en elementos estructurales coherentes (pilares, contrafuertes, muros de carga y tabiques, arcos, bóvedas, etc.), permitiendo una primera reflexión sobre las características constructivas y compositivas del edificio que sirven de guía para el análisis definitivo.

La preparación de una ficha de campo $^{34}$ para la recogida de la mayoría de informaciones de carácter técnico-constructivo es el paso más complejo en el sistema de almacenamiento de datos. Nuestra propuesta (Fig. 1) es el fruto de un largo proceso de modificación de un prototipo muy sencillo, estructurado, en un primer momento, sobre la base de la bibliografía tradicional y adaptado, con amplias y continuas correcciones, a la unicidad de los diferentes panoramas constructivos estudiados. La estructura abierta de la misma permite integrar, continuamente, nuevos elementos a los registros que proponemos y plasmar nuevos sistemas conformes a las exigencias de otros territorios.

Es preciso recordar que el punto de partida del análisis de la técnica constructiva se refiere, en todo caso, a un tipo especifico de estructura que desarrolla en el edificio una función estática determinada. A partir de esta consideración se desarrolla el sistema de registro de la totalidad de sus características formales y funcionales.

En la serie de "datos generales» sobre el edificio estudiado y la referencia a la documentación gráfica asociada, se prioriza la relación entre el tipo de estructura (cimentación, muro de carga y tabique, contrafuerte, pilar, jamba, solución de esquina, escalera, pavimento, arco, bóveda, ventana, canalización) y la técnica analizada, con un breve resumen de la situación estratigráfica en la que se encuentra la estructura y la indicación de las unidades estratigráficas de referencia y la posición relativa con respecto a estructuras adyacentes.

Bajo estas informaciones de carácter general, el sistema de documentación se divide en tres partes:

1. Análisis de características constructivas.

2. Materiales constructivos.

3. Procesos constructivos.

En la primera sección se analizan los tipos de aparejos, evitando, en esta fase, la atribución de la nomenclatura tradicional de las técnicas constructivas romanas, con el objetivo de facilitar los eventuales cambios producidos en

${ }^{34}$ Agradezco a Tomás Cordero su ayuda en la maquetación final de la ficha presentada. el ámbito de una misma clase, en relación con la presencia de posibles variantes territoriales.

En el examen de los tipos de aparejos (Sillería, Sillarejo, Mampostería, Aparejo de ladrillo, Aparejos «de marco", Aparejos mixtos, Otros aparejos) se ha privilegiado la documentación de información vinculada a elementos formales (regularidad de los aparejos, disposición de hiladas) con elementos estructurales (empleo de diferentes tipologías de elementos constructivos, presencia de elementos de nivelación o añadidos a la realización estándar del paramento, empleo de morteros) y tipos de revestimiento en el caso que se conserven.

Se presta particular atención, además, a las características de las juntas obtenidas tras la colocación de los elementos constructivos, indicadores del grado de conocimiento tecnológico alcanzado en la realización de una técnica determinada y posible elemento de reconocimiento de cuadrillas de trabajo entre la mano de obra.

La misma importancia se ha atribuido al tipo de mortero empleado y a las características y componentes del núcleo interno de las estructuras, dado que se ha podido observar que paramentos externos similares, aparentemente pertenecientes a una misma técnica edilicia, presentan núcleos internos completamente distintos que plantean la presencia de mano de obra diferenciada, o elecciones peculiares que es preciso registrar y comprender.

En la sección relativa a los materiales se ha distinguido entre materiales lapídeos y ladrillos. Se trata de una sección dedicada a la documentación de los aspectos productivos vinculados con una determinada técnica. Se prioriza la recopilación de datos sobre la forma de los elementos constructivos, la elaboración, las huellas de las herramientas empleadas, las dimensiones y la presencia eventual de modulaciones constructivas recurrentes.

En el caso de la sillería, se ha prestado atención a la documentación de las características físicas de los almohadillados, con el fin de recopilar, para este tipo de acabado, una serie suficiente de datos procedentes de diferentes territorios, que ayuden a establecer si efectivamente se trata de un indicador cronológico vinculado a un estilo arquitectónico o de un recurso económico en relación con la estandarización de la producción de los elementos constructivos.

En la última sección se propone un breve compendio para documentar los procesos relacionados con la producción de una técnica edilicia específica. La búsqueda de indicadores para la reconstrucción de las dinámicas edilicias de época romana se ha planteado mediante la recopilación de datos materiales que se puedan observar en las estruc- 

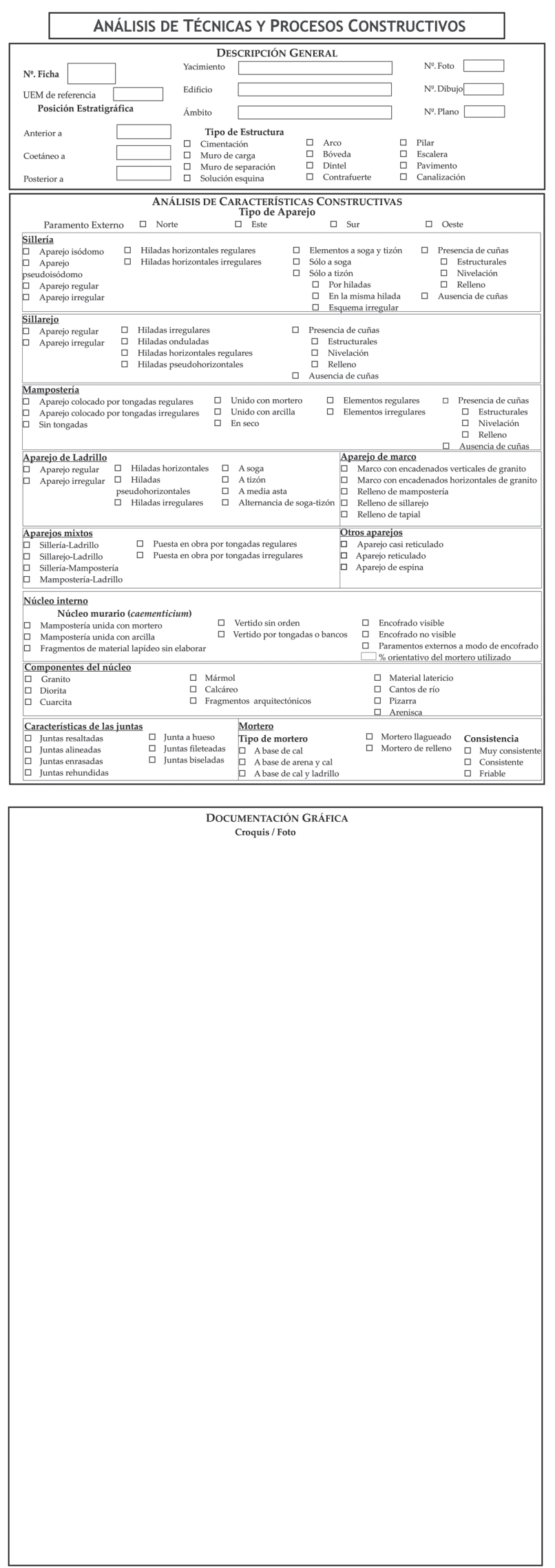

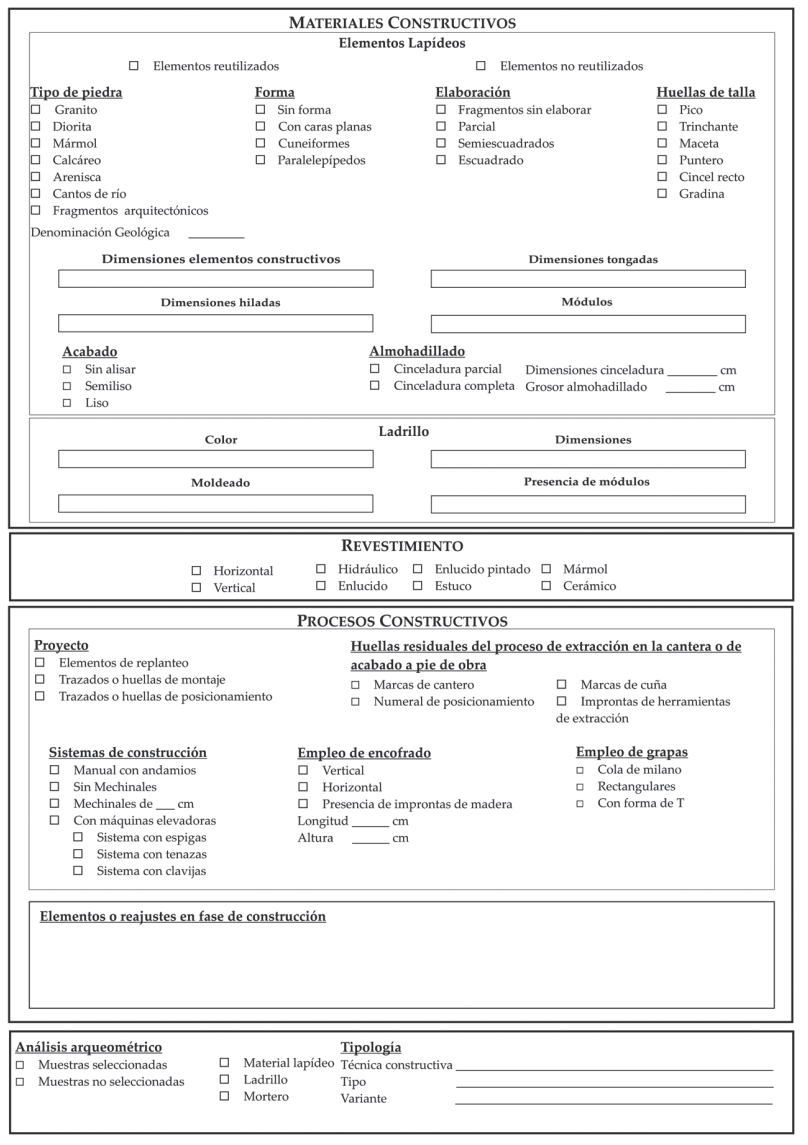

OBSERVACIONES

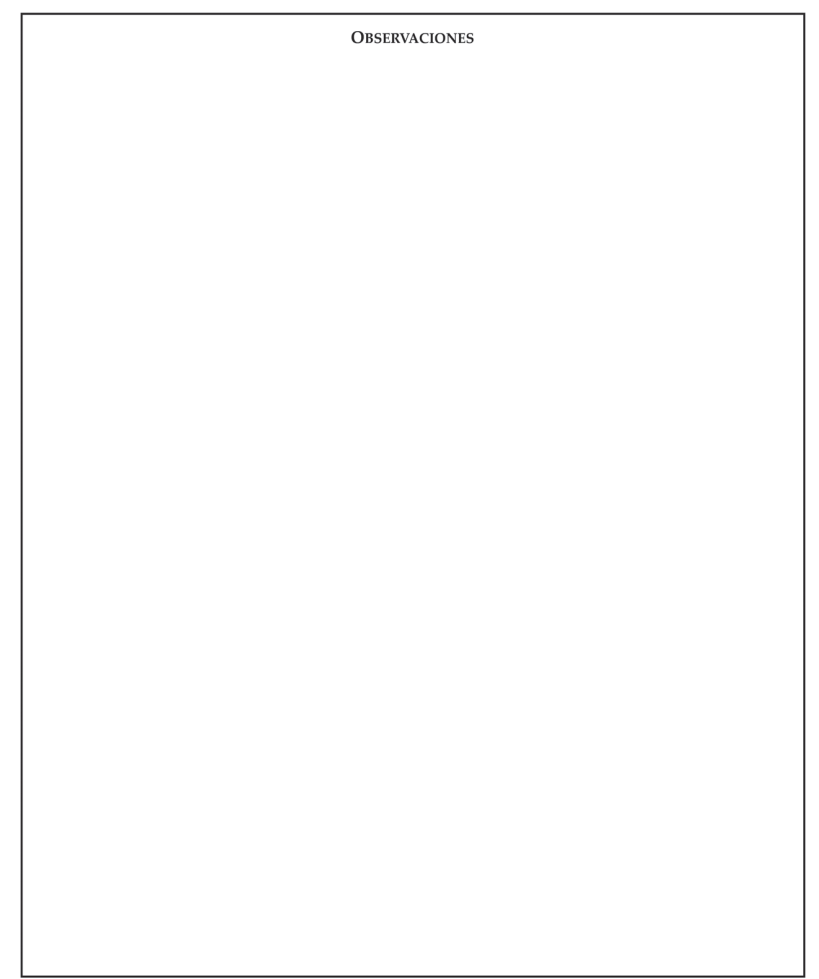

Cronología

Etapa Historica $\square$ Cronologia $\square$

Fecha

Fig. 1. Propuesta de ficha para la documentación de las técnicas constructivas de época romana 
turas sobre el proyecto arquitectónico; huellas residuales del trabajo en la cantera o a pie de obra; distintos sistemas de construcción empleados, variables dependientes de las posibilidades económicas de una obra; uso de distintos tipo de encofrados, grapas, mechinales; y, finalmente, los errores y reajustes que se producen continuamente en una obra y que, escasas veces, se registran. Estos últimos permiten añadir elementos fundamentales para el estudio de la realización de estructuras determinadas y ajustar, en términos relativos, los tiempos de ejecución y finalización del trabajo, a menudo calculados solamente sobre la base de la cronología ofrecida por un epígrafe.

\section{OBJETIVOS DE LA CLASIFICACIÓN DE LAS TÉCNICAS CONSTRUCTIVAS ROMANAS: LA TIPOLOGÍA}

En general, nuestras clasificaciones se han efectuado con el objetivo final de elaborar una cronotipología entre la forma de los aparejos y la función desarrollada en el funcionamiento general de los edificios.

¿Cómo plantear el siguiente paso de la tipología? Con el sistema de clasificación y recopilación de los datos se ha efectuado una operación de descomposición de los edificios, intentando elaborar una cronología relativa entre los tipos de aparejos y los grupos funcionales (cimentaciones, arcos, bóvedas, etc.), teniendo en cuenta que no siempre las semejanzas formales son indicio de coetaneidad, ni las diferencias entre técnicas lo son de distinción cronológica. La clasificación es solamente un punto de partida para estructurar una cronología relativa de un contexto territorial o regional, mediante la concatenación de aspectos técnicos o formales. Se trata de crear un instrumento para reconstruir, a partir del análisis de los aparejos, la formación de una determinada técnica, con sus variaciones debidas al condicionamiento del material constructivo del que se dispone. En este sentido, la referencia al contexto geográfico resulta de gran importancia para el reconocimiento del tipo de material y la forma de aparejarlo en relación con los conocimientos técnicos de los ejecutores materiales de las obras.

El paso siguiente es ordenar la serie de variantes documentadas en una tipología microterritorial comparable con otros sistemas realizados con la misma base de documentación. Con este fin, los datos ofrecidos por las variantes técnicas documentadas pueden indicar elementos claros que caracterizan un determinado contexto territorial.

En nuestra opinión, es posible crear cuatro categorías piramidales que permitan estructurar una tipología correcta: las clases, los grupos, los tipos y las variantes, general- mente en relación con el tipo de material empleado en la construcción de un determinado tipo de aparejo.

Las clases corresponden a las mismas técnicas constructivas que podemos definir, en esta fase, según la nomenclatura tradicional (opus quadratum, incertum, mixtum, testaceum, caementicium, reticulatum etc.), caracterizadas, básicamente, por la relación entre el tipo de material y la técnica empleada.

Los grupos representan una subdivisión muy amplia de las características morfológicas generales asociadas a una determinada técnica constructiva. Se caracterizan por el tipo de material, la presencia y el tipo de mortero, la técnica de los núcleos internos de las estructuras y la morfología de los materiales.

En la definición de los tipos entran una serie de detalles más específicos basados en la talla, elaboración y acabado de los materiales, las dimensiones, la colocación de los elementos constructivos y la presencia de revestimientos. El problema recurrente del análisis de los tipos es la elección de los elementos que vinculan las características discriminantes. El tipo se puede identificar, así, como «una suma de atributos, es decir, de elementos formales definidos previamente o bien como un objeto global, caracterizado por atributos específicos» ${ }^{35}$.

Las variantes, en cambio, constituyen la totalidad de las variaciones formales y técnicas respecto a la categoría superior, el tipo, y atribuyen las características determinantes a este último. Es preferible registrar un amplio abanico de variables, con la intención de no dejar sin documentar posibles elementos que, si en un contexto determinado pueden constituir solamente una pequeña variación a la regla general del tipo, en otro, puede adquirir la entidad de un tipo recurrente.

Para la datación de la tipología es necesario emplear fuentes directas de tipo relativo o absoluto, vinculadas con los niveles de construcción de las estructuras en cuestión o, en casos concretos, con la presencia de epígrafes que, sin embargo, presentan problemas interpretativos causados por la relación estratigráfica entre el soporte y la inscripción o por el tiempo transcurrido entre la construcción de las estructuras y su colocación.

La atención prestada a la cronotipología de las técnicas constructivas, no debería obviar la necesidad de realizar análisis cuantitativos y arqueométricos. En este sentido, se

\footnotetext{
${ }^{35}$ Sestieri, A.M. 2001: p. 62. En realidad, el debate sobre el concepto de tipo es intrínseco al desarrollo mismo de las distintas disciplinas que forman la ciencia arqueológica. Las mejores propuestas para su definición vienen, sin embargo, de la ceramología. Para una definición del concepto de tipo véase también Carandini, A. 1979; Morel, J.P. 1981; Arthur, P. - Ricci, A. 1981: pp. 125-128; Pucci, G. 1983: pp. 273-290; Ricci, A. 1985: pp. 11-19.
} 
recomienda efectuar una recogida de muestras especificas de morteros asociadas al reconocimiento de tipos y variantes para trabajos monográficos sobre la composición de los mismos y la tecnología empleada en la ejecución.

En el estudio de la cultura material no basta con reconocer los materiales y las técnicas empleadas en la construcción de un edificio, sino que es necesario extraer, con la ayuda de los análisis arqueométricos, la procedencia y las características tecnológicas de los mismos, elementos fundamentales para definir los distintos niveles de conocimiento alcanzados en la producción de determinadas formas constructivas o bien la calidad conseguida respecto a otros contextos. Estos detalles sirven, además, para comprender los cambios que se producen en una determinada manera de construir, las innovaciones respecto a la tradición o su mantenimiento y, finalmente, el grado de dependencia de los cambios tecnológicos ya sea de la voluntad de los promotores o de la de arquitectos y constructores $^{36}$.

\section{CONCLUSIONES}

La experiencia llevada a cabo en el estudio de distintos edificios de época romana nos indica que las técnicas no se pueden clasificar solamente sobre la base formal de los paramentos exteriores. No existe, como se ha creído a partir de una mala interpretación de la obra de G. Lugli ${ }^{37}$, un pretendido significado evolutivo de los términos opus siliceum, quadratum, incertum, reticulatum, etc. Es fundamental comprender que las principales clases coexisten entre sí y, consecuentemente, las dinámicas que conducen a que los tipos se implanten, se transformen, se transmitan sin cambios o desaparezcan en un contexto territorial específico.

El objetivo es analizar de forma diacrónica las técnicas constructivas que se distinguen en un área homogénea, desde el punto de vista de los materiales constructivos y las formas de aparejar las estructuras. Se delimita, así, un «área tecnológica». En el interior del «área» es obligatorio reconocer ámbitos técnico-culturales que producen, con procesos específicos, unas determinadas técnicas de las que es necesario encontrar su comienzo y su desarrollo, mediante la distinción del mayor número posible de variables en el ámbito de un mismo grupo técnico.

El conocimiento del «área», posibilitará inevitablemente la comprensión de la cultura arquitectónica presente en un territorio en un momento histórico concreto.

\footnotetext{
${ }^{36}$ Mannoni, T. Boato, A. 2001: p. 44.

${ }^{37}$ Lugli, G. 1957.
}

Dicha cultura arquitectónica no dependerá solamente de la tendencia técnica sino también de los factores geológicos que condicionan el aprovisionamiento, la talla y la elaboración de los materiales constructivos o del ámbito territorial de pertenencia con su relativa homogeneidad cultural, geológica, histórica, económica, etc. ${ }^{38}$

Mediante la composición de un amplio mosaico de regiones diversas, estudiadas con similares planteamientos, se descubrirán las reglas generales comunes y las diferencias entre ciudades, zonas geológicamente homogéneas o provincias. En este sentido, el análisis descriptivo, la clasificación y la consecuente ordenación tipológica de las técnicas, constituyen niveles de la investigación que representan solamente el primer estadio hacia una lectura compleja que atienda a la reconstrucción de las diferentes culturas arquitectónicas del mundo romano.

En el momento en que se alcancen datos relativos de ámbitos territoriales o regionales distintos y se afinen las cronologías de las técnicas constructivas, será posible, quizá, pensar en los motivos que llevaron a la construcción de determinados tipos de edificios con procesos de edificación específicos, en determinados lugares y periodos históricos.

El objetivo de la clasificación de las técnicas constructivas de época romana de acuerdo con los parámetros explicados tiene como finalidad un análisis más general sobre el significado de las distintas unidades arquitectónicas, los edificios, con la intención de reconstruir el equilibrio y la interacción entre los materiales de construcción, entre las estructuras y su función estática o, simplemente, entre las diferentes formas de construir.

Con este objetivo de desarrollo futuro se ha propuesto un sistema de recopilación de informaciones esenciales organizado sobre la documentación de la tradición tecnológica asociada a la construcción romana, con la intención de definir las características específicas del conocimiento de los ejecutores materiales de las obras y de homogeneizar de la mejor manera posible la recopilación de la documentación para el análisis de las técnicas constructivas.

\section{Bibliografía}

Arthur, P. - Ricci, A. 1981: «Sistemi di classificazione della cerámica proveniente da scavi di complessi di epoca romana", Dialoghi di Archeologia, I, pp. 125128.

Azkarate Garai-Olaun, A. 2002: «Intereses cognoscitivos y praxis social en Arqueología de la Arquitectura", Arqueología de la Arquitectura I, 2002, pp. 55-71.

Azkarate, A. - Cámara, L. - Lasagabaster, J.A. - La Torre, P. 2001: Catedral de Santa Maria. Vitoria-Gasteiz. Plan Director de Restauración, Vitoria.

\footnotetext{
${ }^{38}$ Ferrando Cabona, I. 1998: pp. 75-79.
} 
Bendala Galán, M. 1992: «Materiales de construcción romano: peculiaridades de España», Ciencia, metodologias y téenicas aplicadas a la arqueología (Rodá, I. Ed.), pp. 215-226, Barcelona.

Bendala, M. - Durán, R. 1994: «El anfiteatro de Augusta Emerita: rasgos arquitectónicos y problemática urbanística y cronológica, El anfiteatro en la Hispania romana (Coloquio Internacional, Mérida, 26-28 de Noviembre de 1992), pp. 247-264.

Bendala, M. - Rico, C. - Roldán, L. (Eds.) 1998: El ladrillo y sus derivados en la época romana, Madrid.

Brogiolo, G.P. 1996: «Prospettive per l'archeologia dell'architettura», Archeologia dell'Architettura I, 1996, pp.11-15.

Brogiolo; G.P. - Zonca, A. 1989: «Residenze medievali (XI-XII secc.) nel territorio lombardo", Storia della città, 52, pp. 37-44.

Caballero Zoreda, L.1987: «El método arqueológico en la comprensión del edificio. (Sustrato y estructura)", Curso de Mecánica y tecnología de los edificios antiguos, Colegio Oficial de Arquitectos de Madrid, pp. 13-58.

Caballero Zoreda, L. 1996: «El análisis estratigráfico de construcciones históricas", Arqueología de la Arquitectura. El método arqueológico aplicado al proceso de estudio y de intervención en edificios históricos (Caballero Zoreda, L. Escribano Velasco, C. Eds.), pp. 55-74, Burgos.

Caballero Zoreda, L. 1999: «Aportación a la arquitectura medieval española. Definición de un grupo de iglesias castellanas, riojanas y vascas», Actas del V Congreso de Arqueología Medieval Española (Valladolid), 1, pp. 221233.

Caballero Zoreda, L. - Escribano Velasco, C. 1996 (Eds.): Arqueología de la Arquitectura. El método arqueológico aplicado al proceso de estudio y de intervención en edificios históricos, Burgos.

Caballero Zoreda, L. - Fernández Mier, M. 1997: «Análisis arqueológico de construcciones históricas en España. Estado de la cuestión", Archeologia dell'Architettura II, 1997, L'archeologia del costruito in Italia e in Europa. Esperienze a confronto e orientamenti della ricerca (Atti della Giornata di Studi, Genova 10 Maggio 1996), pp. 147-158.

Cagnana, A. 1994: «Archeologia della produzione fra tardo-antico e altomedioevo: le tecniche murarie e l'organizzazione dei cantieri», Edilizia residenziale tra Ve VIII secolo (Brogiolo, G.P. Ed), pp. 39-52, Mantova.

Camporeale, S. 2004: «Tecniche edilizie in pietra nella Mauretania Tingitana tra l'epoca mauretana e romana. Osservazioni sulle apparecchiature e utilizzo della malta», Archeologia dell'Architettura IV, 2004, pp. 195-205.

Carandini, A. 1979: Archeologia e cultura materiale, Bari.

Durán Cabello, R. 1991-1992: «Técnicas de edilicia romana en Mérida I», Anas, 4/5, pp. 45-80, Mérida.

Durán Cabello, R. 2004: El teatro y el anfiteatro de Augusta Emerita. Contribución al conocimiento histórico de la capital de Lusitania, BAR Internacional Series 1207, Oxford.

Ferrando Cabona, I. 1998: «Problemi di datazione in archeologia dell'architettura», Archeologia dell'Architettura III, 1998, pp. 75-79.

Gardin, J.C. 1979: Une archéologie théorique, Paris.

Lugli, G. 1957: La tecnica edilizia romana, Roma.

Mannoni, T. 1974: «Lanalisi delle tecniche murarie medievali in Liguria», Atti del colloquio internazionale di Archeologia Medievale, pp. 291-300, Erice 1974, Palermo 1976.
Mannoni, T. 1984: «Metodi di datazaione dell'edilizia storica», Archeologia Medievale XI, 1984, pp. 396-403.

Mannoni, T. 1994: Caractteri costruttivi dell'edilizia storica, Genova.

Mannoni, T. 1997: «Il problema complesso delle murature storiche in pietra. 1. Cultura materiale e cronotipología», Archeologia dell'Architettura II, 1997, pp. 15-24.

Mannoni, T. - Giannichedda, E. 1996: Archeologia della produzione, Torino.

Mannoni, T. Boato, A. 2002: «Archeologia e storia del cantiere di costruzione», Arqueología de la Arquitectura I, 2002, pp. 39-53.

Morel, J.P. 1981: Céramique campanienne. Les formes, Roma.

Pais, A. 2008: «L'edilizia romana nella toscana tirrenica alla luce dell’archeologia dell'architettura». En Camporeale, S. - Dessales, H. - Pizzo, A. (eds.) 2008: Arqueología de la Construcción I. Los procesos constructivos en el mundo romano: Italia provincias y occidentales, (Mérida, Instituto de Arqueología 25-26 de Octubre de 2008), Anejos de Archivo Español de Arqueología no 50, pp. 6788 , Madrid.

Parenti, R. 1988a: «Le tecniche di documentazione per una lettura stratigrafica dell'elevato", Archeologia e restauro dei monumenti (Francovich, R. - Parenti, R. 1988 Eds.), pp. 249-279, Firenze.

Parenti, R. 1988b: «Sulle possibilità di datazione e di classificazione delle murature», Archeologia e Restauro dei monumenti (Pontignano 1987), R. Francovich - R. Parenti (Eds.), Firenze 1988, pp. 280-304.

Parenti, R. 1994: «I materiali da costruzione, le tecniche di lavorazione e gli attrezzi», Edilizia residénciale tra Ve VIII secolo (Brogiolo, G.P. Ed.), pp. 25-37.

Pizzo, A. 2003: Reseña a «Catedral de Santa Maria. Vitoria-Gasteiz. Plan Director de Restauración. Azkarate, A. - Cámara, L. - Lasagabaster, J.A. - La Torre, P., Archivo Español de Arqueología, 76, 2003, no 187-188, pp. 316-317.

Pizzo, A. 2010: Las técnicas constructivas de la arquitectura pública de Augusta Emerita, Anejos de AEspA 56, Madrid.

Pucci, G. 1983: «Cerámica, tipi, segni», Opus II, 1983, pp. 273-290.

Ricci, A. 1985: «Introduzione», Settefinestre. Una villa schiavistica nell'Etruria romana, Vol. III. La villa e i suoi reperti (Ricci, A. Ed.) pp. 11-19, Modena.

Rodríguez Gutiérrez, O. 2004: El teatro romano de Itálica. Estudio arqueoarquitectónico, Madrid.

Roldán Gómez, L. 1987: «Técnica edilicia en Itálica. Los edificios públicos», Archivo Español de Arqueologia, 60, 1987, pp. 89-122.

Roldán Gómez, L. 1992: Técnicas constructivas romanas en Carteia (San Roque, Cadiz), Madrid.

Roldán Gómez: L. 1994a: Técnicas constructivas romanas en Italica, Madrid.

Sánchez Zufiaurre, L. 2007: Técnicas constructivas medievales. Nuevos documentos arqueológicos para el estudio de la Alta Edad Media en Álava, Vitoria.

Sestieri, A.M. 2001: "Clasificación y tipología», Diccionario de Arqueología (Francovich, R. - Manacorda, R. 2001 Eds.), pp. 61-66, Barcelona.

Utrero Agudo, M.Á. 2008: «Arqueología de la Arquitectura y patrimonio edificado. Experiencias y perspectivas una década después». En La investigación sobre Patrimonio Cultural (C. Sáiz Jiménez - M.Á. Rogelio Candelero eds.) Sevilla.

Recibido: 14 de septiembre de 2010 Aceptado: 8 de noviembre de 2010 